\title{
Microsurgical Gross Total Resection of Foramen Magnum Meningioma via Far Lateral Approach
}

\author{
Sima Sayyahmelli ${ }^{1}$ Mustafa K. Başkaya ${ }^{1}$ \\ ${ }^{1}$ Department of Neurological Surgery, University of Wisconsin \\ Medical School, Madison, Wisconsin, United States \\ J Neurol Surg B 2019;80(suppl S4):S360-S362.
}

\begin{abstract}
Address for correspondence Mustafa K. Başkaya, MD, Department of Neurological Surgery, University of Wisconsin Medical School, K4/834 CSC, 600 Highland Avenue, Madison, WI 53792-0001, United States (e-mail: baskaya@neurosurgery.wisc.edu).
\end{abstract}

\begin{abstract}
Keywords

- far lateral transcondylar

- foramen magnum meningioma

- gross total resection

- skull base

Foramen magnum meningiomas are one of the most challenging tumors for skull base neurosurgeons due to their proximity to critical neurovascular structures. The farlateral approach demonstrated here facilitates access to lesions involving the anterior portion of the foramen magnum.

In this video, we present a 62 -year-old woman with hand numbness and weakness. The patient had significant difficulty in fine motor movements of both hands. In the neurological examination, she had a significant right-hand intrinsic muscle weakness and mild quadriparesis.

Magnetic resonance imaging (MRI) showed a dural-based homogeneously enhancing extra-axial mass in the anterior foramen magnum with a significant mass effect on the brain stem and the upper cervical cord. The decision was made to proceed with a far lateral transcondylar skull base approach including partial C1 laminectomy.

The surgery and postoperative course were uneventful. The postoperative MRI showed gross total resection of the mass. The histopathology indicated a WHO (World health Organization) grade-I meningioma. The patient's postoperative course was uneventful. She improved to normal neurological function within several weeks and continues to do well without recurrence at 20 months' follow-up.

In this video, we demonstrated important steps for the microsurgical resection of these challenging lesions.

The link to the video can be found at: https://youtu.be/_nuX2Y7YU9w.
\end{abstract}

\section{Disclosure of Funding}

None.

Conflict of Interest

None.

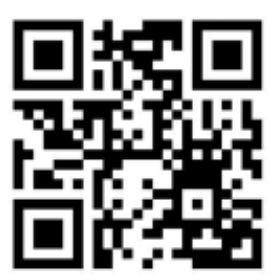

received

February 15, 2019

accepted

July 9, 2019

published online

October 15, 2019

www.thieme.com/skullbasevideos

www.thieme.com/jnlsbvideos

DOI https://doi.org/

10.1055/s-0039-1695063.

ISSN 2193-6331. (c) 2019 Georg Thieme Verlag KG Stuttgart · New York
License terms

c) $(1) \$$ 


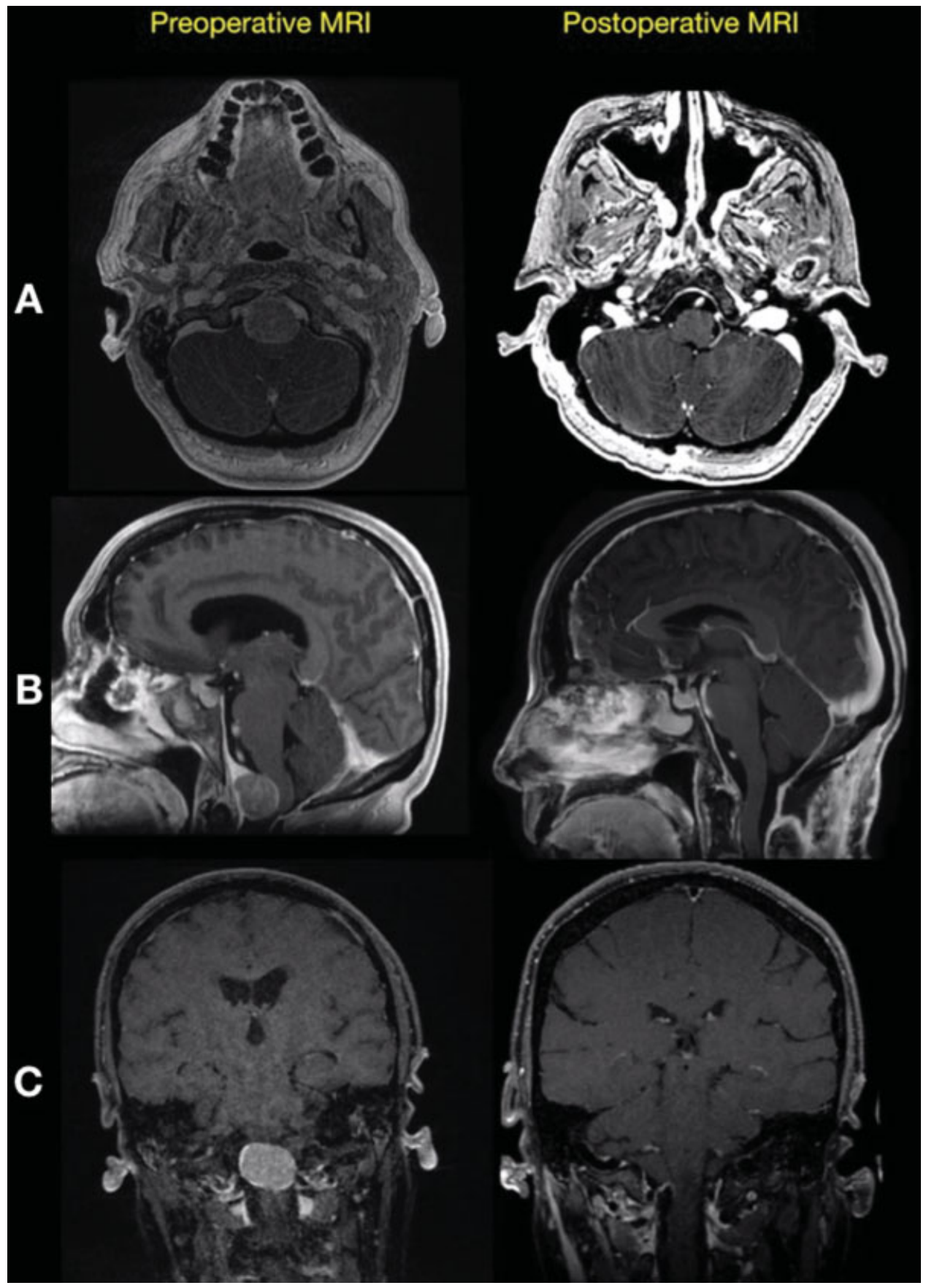

Fig. 1 Preoperative MRI in (A) axial, (B) sagittal, and (C) coronal planes shows a dural-based heterogeneous enhancing extra-axial mass in the anterior foramen magnum with a significant mass effect upon the upper cervical cord and postoperative MRI in (A) axial, (B) sagittal, and (C) coronal planes shows a gross total resection of the tumor without recurrence. MRI, magnetic resonance iaging. 


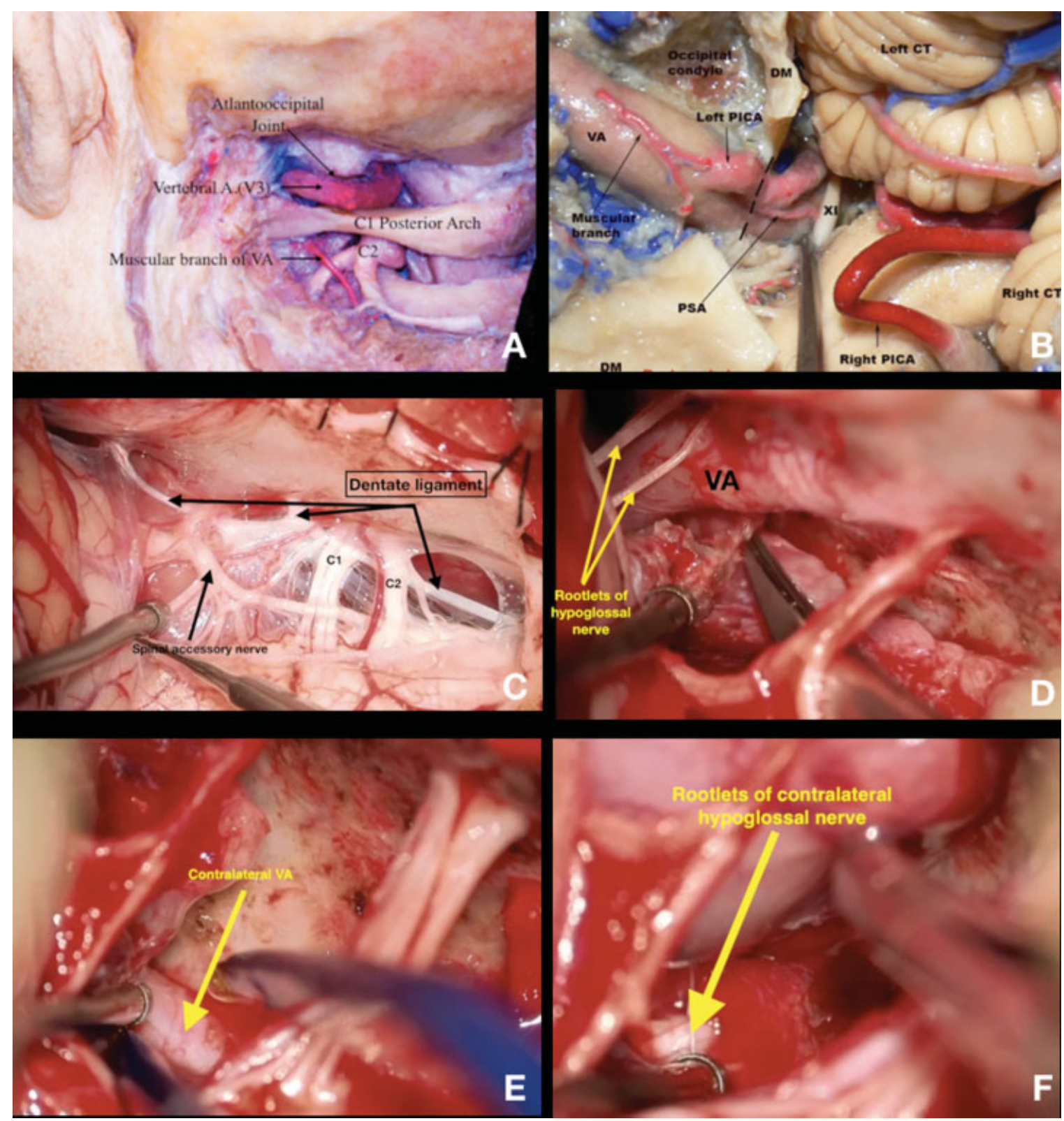

Fig. 2 (A) The cadaveric dissections of far lateral approach from our laboratory have demonstrated the extent of access and related anatomic and neurovascular structures via this approach. (B) The cadaveric dissections from our laboratory shows the vertebral artery and the posterior inferior cerebellar artery were exposed. In this approach for the foramen magnum meningioma, only minimal drilling of the occipital condyle is necessary. (C) The intraoperative picture shows the dentate ligament, C1 and C2 nerve roots, and the accessory nerve after dural opening. (D) The intraoperative picture shows the ipsilateral vertebral artery and the rootlets of the hypoglossal nerve. (E) The intraoperative picture shows the contralateral vertebral artery. (F) The intraoperative picture shows the rootlets of the contralateral hypoglossal nerve. DM, dura mater; PICA, posterior inferior cerebellar artery; VA, vertebral artery; PSA, posterior spinal artery. 